\title{
A New Approach for Guaranteed State Estimation by Zonotopes
}

\author{
V.T.H. Le* T. Alamo** E.F. Camacho ${ }^{* *}$ C. Stoica* \\ D. Dumur* \\ * SUPELEC Systems Sciences (E3S) - Automatic Control Department, \\ 3 rue Joliot Curie, F-91190, Gif-sur-Yvette cedex, France \\ (e-mail: \{vutuanhieu.le,cristina.stoica,didier.dumur\}@supelec.fr). \\ ** Department of Ingeniería de Sistemas y Automática, Universidad de \\ Sevilla, Camino de los Descubrimientos, 41092 Sevilla, Spain \\ (e-mail: alamo@cartuja.us.es; eduardo@esi.us.es).
}

\begin{abstract}
This paper proposes a methodology for guaranteed state estimation of linear discretetime systems in the presence of bounded disturbances and noises. This aims at computing an outer approximation of the state estimation domain represented by a zonotope. A new criterion is used to reduce the size of the zonotope at each sample time. An illustrative example is analyzed in order to highlight the advantages of the proposed algorithm.
\end{abstract}

Keywords: Set-membership estimation, system state estimation, zonotopes, bounded noises, LMI.

\section{INTRODUCTION}

The problem of state estimation of uncertain systems has received increasing attention from researchers over the last years. This problem may be synthesized as follows: given a mathematical model of a real system and allowed some measurements, the state of the real system has to be estimated. This is generally difficult and time consuming as the mathematical model of a real system is never a perfect representation of the existing plant and the measurements do not provide perfect data due to existing noises. There are several approaches in the literature which deal with this problem such as stochastic approaches (Kalman filter theory) and the set-membership estimation Schweppe (1968), Bertsekas and Rhodes (1971), Walter and Piet-Lahanier (1989), Durieu et al. (2001), Combastel (2003) and Alamo et al. (2005).

The Kalman filter (Kalman (1960), Sorenson (1983)) combines all available measurement data and the knowledge of the system to provide an estimate of the desired variable by minimizing the error variance. This approach considers white and Gaussian distribution of the random variables of the model, with the inconvenience that these assumptions could be sometimes difficult to validate.

The set-membership estimation has been developed in the last 35 years (Schweppe (1968)). This method relies on the description of uncertainties belonging to bounded compact sets. The state estimation set is a compact set containing all possible states of the system that are consistent with the uncertain model and the measurement noise. No other hypotheses on the distribution of the perturbation are assumed. Different domain representations can be used for the estimation set as ellipsoids Schweppe (1968), Bertsekas and Rhodes (1971), and Durieu et al. (2001), polytopes (boxes, parallelotopes) Walter and Piet-Lahanier (1989).
When the set-membership estimation is used, there is a trade-off between the computation load and the set size. Polytopes can be used for an exact representation of the domains of the system state in a linear formulation. However efficient results may be obtained only for a reasonable number of vertices of the polytopes Walter and PietLahanier (1989). To overcome this drawback, the representation by ellipsoids has been firstly used, sometimes with a significant loss of performance. In the recent years zonotopes (a particular class of polytopes) have received more attention because of their advantages in comparison with ellipsoids Vicino and Zappa (1996), Kühn (1998), Lalami (2008). Contrary to ellipsoids, the Minkowski sum of two zonotopes is a zonotope, this property being very useful in the prediction step of state estimation. Zonotopes can represent uncertainties due to independent disturbances in each direction of the state-space and zonotopes are a suitable representation for controlling the wrapping effect Kühn (1998). The domain representation by zonotopes is used for many applications such as: the reachability analysis (Althoff et al. (2007)), collision detection (Guibas et al. (2005)) and states estimation (Combastel (2003), Alamo et al. (2005)). To estimate the system state, in Combastel (2003) a singular-value decomposition is used in the correction step to obtain an outer approximation of the intersection between the uncertain trajectory and the region of the state space that is consistent with the measured output. In Alamo et al. (2005), the outer approximation is elaborated as a family of zonotopes, parameterized by a free vector. This vector is computed by solving different optimization problems such as segment minimization or volume minimization. The segment minimization offers a fast computation time but the obtained domain can be too large (and hence the results can be conservative). The volume minimization has a longer computation time and better performance but it can lead to a very narrow zono- 
tope (i.e. the uncertainty in some directions can remain extremely large even when the volume of the zonotope tends to zero). This motivates the use of a different optimization criterion that should be an acceptable trade-off between these two criteria.

In this paper, a new method for guaranteed state estimation in the case of linear discrete-time system with bounded disturbances and measurement noises is presented. This method is proposed to solve these weakpoints of the existing methods. A new criterion is used in the correction step to obtain a better result both for the performance and computation time of state estimation. For the previous methods, some computations were performed during the correction step (e.g. singular value decomposition in Combastel (2003), optimization problem in Alamo et al. (2005)). The new method allows to perform these computations off-line and this is a major advantage for real time applications. Moreover, a new criterion on the radius of the zonotope is presented to solve the problem of a very narrow zonotope in the volume minimization algorithm.

The paper is organized as follows. Section 2 presents useful mathematical notations and basic definitions. In section 3 , the class of uncertain dynamical systems used along this paper is defined. The next section presents a new approach to compute an outer bound of the state estimation by zonotopes. In Section 5 an example is proposed to show the advantages of the developed algorithm. Finally, some concluding remarks and future work are presented.

\section{MATHEMATICAL NOTATIONS AND BASIC DEFINITIONS}

An interval $[a ; b]$ is defined as the set $\{x: a \leq x \leq b\}$. The unitary interval is $\mathbf{B}=[-1 ; 1]$.

A box $\left(\left[a_{1} ; b_{1}\right], \ldots,\left[a_{n} ; b_{n}\right]\right)^{T}$ is an interval vector. A unitary box in $\mathbb{R}^{m}$, denoted $\mathbf{B}^{m}$, is a box composed by $m$ unitary intervals.

The Minkowski sum of two sets $X$ and $Y$ is defined by $X \oplus Y=\{x+y: x \in X, y \in Y\}$.

A polytope $\Omega$ is the convex hull of its vertices

$\Omega=C o\left\{v_{1} ; v_{2} ; \ldots ; v_{n}\right\}$. This means that if $v \in \Omega$ then $v=\sum_{i=1}^{n} \alpha_{i} v_{i}$ with $\sum_{i=1}^{n} \alpha_{i}=1$ and $\alpha_{i} \geq 0$ for $i=1, \ldots, n$.

Zonotopes are a special class of convex polytopes. A $m$-zonotope in $\mathbb{R}^{n}$ can be defined as the linear image of a $m$-dimensional hypercube in $\mathbb{R}^{n}$. Given a vector $p \in \mathbb{R}^{n}$ and a matrix $H \in \mathbb{R}^{n \times m}$ a $m$-zonotope is the set: $p \oplus H \mathbf{B}^{m}=\left\{p+H z, z \in \mathbf{B}^{m}\right\}$. This is the Minkowski sum of the $m$-segments defined by $m$ columns of matrix $H$ in $\mathbb{R}^{n}$.

The $P$-radius of a zonotope $X=p \oplus H \mathbf{B}^{m}$ is defined as $d(x)=\max \left(\|x-p\|_{P}^{2}\right), x \in X$. This notion is related to the ellipsoid $(x-p)^{T} P(x-p) \leq 1$.

Figure (1) shows a zonotope constructed by a linear image of a centered cube $\left(p=\left[\begin{array}{l}0 \\ 0\end{array}\right]\right)$ in $\mathbb{R}^{2}$, with $H=\left[\begin{array}{lll}1 & 2 & 3 \\ 3 & 2 & 1\end{array}\right]$. This figure is obtained by using the indications in Schön and Kutterer (2005).

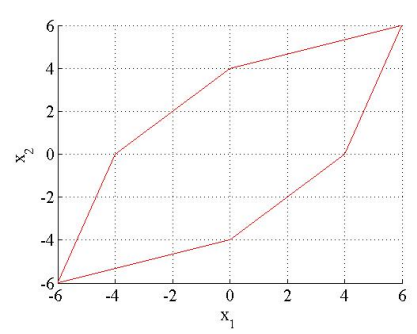

Fig. 1. Construction of a zonotope by a linear transformation

A strip $X$ is defined as the set $\left\{x \in \mathbb{R}^{n}:\left|c^{T} x-d\right| \leq \sigma\right\}$ with $c \in \mathbb{R}^{n}, d$ and $\sigma \in \mathbb{R}$.

A matrix $M=M^{T} \in \mathbb{R}^{n \times n}$ is called a positive-definite matrix (respectively negative-definite matrix), denoted $M \succeq 0(M \preceq 0)$, if $z^{T} M z \geq 0\left(z^{T} M z \leq 0\right)$ for all non-zero vectors $z$ with real entries $\left(z \in \mathbb{R}^{n}\right)$.

Property 1: (Combastel (2003)) Given two centered zonotopes $Z_{1}=H_{1} \mathbf{B}^{m_{1}} \in \mathbb{R}^{n}$ and $Z_{2}=H_{2} \mathbf{B}^{m_{2}} \in \mathbb{R}^{n}$. The Minkowski sum of two zonotopes is also a zonotope defined by $Z=Z_{1} \oplus Z_{2}=\left[\begin{array}{ll}H_{1} & H_{2}\end{array}\right] \mathbf{B}^{m_{1}+m_{2}}$.

Property 2: (Combastel (2003)) The image of a centered zonotope $Z_{1}=H_{1} \mathbf{B}^{m_{1}} \in \mathbb{R}^{n}$ by a linear application $K$ can be computed by a standard matrix product $K \cdot Z_{1}=\left(K \cdot H_{1}\right) \mathbf{B}^{m_{1}}$.

Property 3: (Zonotope reduction) (Combastel (2003), Alamo et al. (2005)) Given a zonotope $Z=p \oplus H \mathbf{B}^{m} \in \mathbb{R}^{n}$ and the integer $s$ with $n<s<m$, denote $\hat{H}$ the matrix resulting from the reordering of the columns of the matrix $H$ in decreasing order of Euclidean norm $\left(\hat{H}=\left[\hat{h}_{1} \ldots \hat{h}_{i} \ldots \hat{h}_{m}\right]\right.$ with $\left.\left\|\hat{h}_{i}\right\|_{2} \geq\left\|\hat{h}_{i+1}\right\|_{2}\right)$. Then $Z \subseteq p \oplus\left[\hat{H}_{T} Q\right] \mathbf{B}^{s}$, where $\hat{H}_{T}$ is obtained from the first $s-n$ columns of matrix $\hat{H}$ and $Q \in \mathbb{R}^{n \times n}$ is a diagonal matrix that satisfies: $Q_{i i}=\sum_{j=s-n+1}^{m}\left|\hat{H}_{i j}\right|$, with $i=1, \ldots, n$.

This property is used in the next section allowing to estimate a high-order zonotope by a lower-order zonotope.

\section{PROBLEM FORMULATION}

Consider the following linear discrete-time invariant system of the form:

$$
\left\{\begin{array}{l}
x_{k+1}=A x_{k}+F \omega_{k} \\
y_{k}=c^{T} x_{k}+\sigma v_{k}
\end{array}\right.
$$

where $x_{k} \in \mathbb{R}^{n}$ is the state of the system, $y_{k} \in \mathbb{R}$ is the measured output at sample time $k$. The vector $\omega_{k} \in \mathbb{R}^{n_{\omega}}$ represents the state perturbation vector and $v_{k} \in \mathbb{R}$ is the measurement perturbation (noise, offset, etc...). It is assumed that the uncertainties and the initial state are bounded by zonotopes: $\omega_{k} \in W, v_{k} \in V$ and $x_{0} \in X_{0}$ ( $W$ and $V$ are assumed containing the origin). $W$ and $V$ are assumed to be unitary boxes. With these notations, the consistent state set and the exact uncertain set are defined as follows.

Definition 1: Given the system (1) and a measured output $y_{k}$, the consistent state set at time $k$ is defined as $X_{y_{k}}=\left\{x \in \mathbb{R}^{n}:\left|c^{T} x-y_{k}\right| \leq \sigma\right\}$. 
Definition 2: Consider the system (1). The exact uncertain state set $X_{k}$ is equal to the set of states that are consistent with the measured output and the initial state set $X_{0}$ : $X_{k}=\left(A X_{k-1} \oplus F W\right) \cap X_{y_{k}}, k \geq 1$.

Remark 1: The exact computation of this set is difficult. To reduce the complexity of the computations, these sets are bounded by means of conservative outer bounds. This paper presents a new method to compute an outer approximation using a zonotope-based procedure. Let us consider that an outer bound of the exact uncertain state set denoted $\hat{X}_{k-1}$ is available at time instant $k-1$. Suppose also that a measured output $y_{k}$ is obtained at time instant $k$. Under these assumptions, an outer bound of the exact uncertain state set can be estimated using the following algorithm.

\section{Algorithm 1}

(1) (Prediction step) Given the system (1), compute a zonotope $\bar{X}_{k}$ that offers a bound for the uncertain trajectory of the system $\left(\bar{X}_{k}=A \hat{X}_{k-1} \oplus F W\right)$.

(2) (Measurement) Compute the consistent state set $X_{y_{k}}$ by using the measurement. According to the assumption on $v_{k}$ this set can be represented by a strip as $\left\{x \in \mathbb{R}^{n}:\left|c^{T} x-y_{k}\right| \leq \sigma\right\}$.

(3) (Correction step) To find the state estimation set, compute an outer approximation $\hat{X}_{k}$ of the intersection between $X_{y_{k}}$ and $\bar{X}_{k}$.

The proposed algorithm is similar to the Kalman filter: the first step is a prediction step while the second and third steps constitute a correction step. To obtain a zonotope bounding the uncertain trajectory of system, the Properties 1 and 2 are used. The complexity of this zonotope is limited by using the Property 3. To compute the intersection set of step 3 an optimization problem is detailed in the next section.

\section{GUARANTEED STATE INTERSECTION}

This section states the main result of this paper. The aim is to find a zonotope that contains the intersection of the two sets $X_{y_{k}}$ and $\bar{X}_{k}$ used by the previous algorithm. As $\bar{X}_{k}$ is a zonotope and $X_{y_{k}}$ is a strip, it is convenient to obtain an outer bound of the intersection of a zonotope and a strip.

The next property provides a family of zonotopes (parameterized by the vector $\lambda$ ) that contains the intersection of a zonotope and a strip.

Property 4: (Alamo et al. (2005)). Given the zonotope $X=$ $p \oplus H \mathbf{B}^{r} \subset \mathbb{R}^{n}$, the strip $S=\left\{x \in \mathbb{R}^{n}:\left|c^{T} x-d\right| \leq \sigma\right\}$ and the vector $\lambda \in \mathbb{R}^{n}$, define a vector $\hat{p}(\lambda)=p+\lambda\left(d-c^{T} p\right) \in$ $\mathbb{R}^{n}$ and a matrix $\hat{H}(\lambda)=\left[\left(I-\lambda c^{T}\right) H \sigma \lambda\right] \in \mathbb{R}^{n \times(m+1)}$. Then the following expression holds $X \cap S \subseteq \hat{X}(\lambda)=$ $\hat{p}(\lambda) \oplus \hat{H}(\lambda) B^{r+1}$.

Proof: This property is proved in Alamo et al. (2005) but to facilitate the reading of this paper the proof is reminded below.

Supposing an element $x \in X \cap S$, on one hand this means that $x \in X=p \oplus H \mathbf{B}^{r}$. Using the definition of a $m$-zonotope implies that there exists a vector $z \in \mathbf{B}^{r}$ such that

$$
x=p+H z
$$

Adding and subtracting $\lambda c^{T} H z$ to the previous equality leads to the following expression:

$$
x=p+\lambda c^{T} H z+\left(I-\lambda c^{T}\right) H z
$$

On the other hand, from $x \in X \cap S$ it is inferred that $x \in S=\left\{x \in \mathbb{R}^{n}:\left|c^{T} x-d\right| \leq \sigma\right\}$. Thus, there exists an unitary interval $\omega \in[-1 ; 1]$ such that $c^{T} x-d=\sigma \omega$. Taking into account the form of the vector $x$ given by (2) leads to $c^{T}(p+H z)-d=\sigma \omega$, which is equivalent to $c^{T} H z=d-c^{T} p+\sigma \omega$. Substituting $c^{T} H z$ in equation (3), the following expression is obtained:

$$
\begin{aligned}
x & =p+\lambda\left(d-c^{T} p+\sigma \omega\right)+\left(I-\lambda c^{T}\right) H z \\
& =p+\lambda\left(d-c^{T} p\right)+\lambda \sigma \omega+\left(I-\lambda c^{T}\right) H z
\end{aligned}
$$

After simple computations and using the notation defined in Property 4, the following form is obtained:

$$
x=\hat{p}(\lambda)+\left[\left(I-\lambda c^{T}\right) H \quad \sigma \lambda\right]\left[\begin{array}{c}
z \\
\omega
\end{array}\right]=\hat{p}(\lambda) \oplus \hat{H}(\lambda)\left[\begin{array}{c}
z \\
\omega
\end{array}\right]
$$

and the following inclusion holds:

$$
x=\hat{p}(\lambda) \oplus \hat{H}(\lambda)\left[\begin{array}{c}
z \\
\omega
\end{array}\right] \in \hat{p}(\lambda) \oplus \hat{H}(\lambda) \mathbf{B}^{r+1}=\hat{X}(\lambda) .
$$

To choose $\lambda$, two approaches were presented in Alamo et al. (2005):

- Minimizing the segments of the zonotope offers a fast computation but with a loss of performance for the estimation;

- Minimizing the volume of the intersection leads to more accurate results, but at each sample time an optimization problem must be solved, which implies the need for a fast real time application.

In this section, a new approach (which is the main contribution of this paper) that offers both good performance and a fast computation time is proposed. This corresponds in fact to a new method used during the correction step of the algorithm proposed in Section 3. A different criterion is presented to compute the vector $\lambda$ to overcome the drawbacks of the two mentioned methods.

Supposing an outer approximation of the state set at the time instant $k$ is $\hat{X}_{k}=p \oplus H \mathbf{B}^{r}$ and the measured output at the instant $k+1$ is $d=y_{k+1}$. The predicted state set at the next instant $\bar{X}_{k+1}$ can be computed using (1), Property 1 and Property 2:

$$
\bar{X}_{k+1}=A p \oplus[A H \quad F] \mathbf{B}^{r+n_{\omega}}
$$

The results stated by Property 4 allow the computation of an outer approximation of the intersection (exact estimation set) between the predicted state set and the strip (which represents the measured output):

$$
\hat{X}(\lambda)=\hat{p}(\lambda) \oplus \hat{H}(\lambda) \mathbf{B}^{r+n_{\omega}+1}
$$

with $\hat{p}(\lambda)=A p+\lambda\left(d-c^{T} A p\right)$

and $\hat{H}(\lambda)=\left[\left(I-\lambda c^{T}\right)[A H F] \sigma \lambda\right]$.

To compute the vector $\lambda$, the approach considered in this paper is the following: compute a symmetric positive definite matrix $P$ and a vector $\lambda$ such that at each sample 
time, the $P$-radius of the zonotopic state estimation set is decreased. This condition can be expressed in a mathematical formulation as follows:

$$
\max _{\hat{z}}\|\hat{H} \hat{z}\|_{P}^{2} \leq \max _{z} \beta\|H z\|_{P}^{2}+\max _{\omega}\|F \omega\|_{2}^{2}+\sigma^{2}
$$

with $\hat{z}=\left[\begin{array}{c}\tilde{z} \\ \tilde{\omega} \\ \eta\end{array}\right] \in \mathbf{B}^{r+n_{\omega}+1}, \tilde{z}, z \in \mathbf{B}^{r}, \tilde{\omega}, \omega \in \mathbf{B}^{n_{\omega}}, \eta \in \mathbf{B}^{1}$, and $\beta \in[0 ; 1)$.

Using the definition of $\hat{H}$ in (8) leads to:

$$
\hat{H} \hat{z}=\left(I-\lambda c^{T}\right)(A H \tilde{z}+F \tilde{\omega})+\sigma \lambda \eta
$$

Using the explicit form of $\hat{z}$, if the following expression is true then the expression (9) is also true:

$$
\max _{\tilde{z}, \tilde{\omega}, \eta}\left(\left\|\hat{H}\left[\begin{array}{c}
\tilde{z} \\
\tilde{\omega} \\
\eta
\end{array}\right]\right\|_{P}^{2}-\beta\|H \tilde{z}\|_{P}^{2}-\|F \tilde{\omega}\|_{2}^{2}-\sigma^{2}\right) \leq 0
$$

which is equivalent to:

$$
\hat{z}^{T} \hat{H}^{T} P \hat{H} \hat{z}-\beta \tilde{z}^{T} H^{T} P H \tilde{z}-\tilde{\omega}^{T} F^{T} F \omega-\sigma^{2} \leq 0
$$

Using the definition $\eta \in \mathbf{B}^{1}$ so $\|\eta\|_{\infty} \leq 1$, the following expression is obtained: $\sigma^{2}\left(1-\eta^{2}\right) \geq 0$. Adding this term to the left of (12) leads to the following sufficient condition for (12):

$$
\begin{array}{r}
\hat{z}^{T} \hat{H}^{T} P \hat{H} \hat{z}-\beta \tilde{z}^{T} H^{T} P H \tilde{z}-\tilde{\omega}^{T} F^{T} F \tilde{\omega}-\sigma^{2}+ \\
+\sigma^{2}\left(1-\eta^{2}\right) \leq 0
\end{array}
$$

Denoting $v=H \tilde{z}$, then the inequality (13) can be written in the matrix formulation:

$$
\left[\begin{array}{c}
v \\
\tilde{\omega} \\
\eta
\end{array}\right]^{T}\left[\begin{array}{ccc}
A_{11} & A_{12} & A_{13} \\
* & A_{22} & A_{23} \\
* & * & A_{33}
\end{array}\right]\left[\begin{array}{c}
v \\
\tilde{\omega} \\
\eta
\end{array}\right] \leq 0
$$

with ${ }^{\prime} *$, denoting the terms required for the symmetry of the matrix and the following additional notations:

$$
\left\{\begin{array}{l}
A_{11}=\left(\left(I-\lambda c^{T}\right) A\right)^{T} P\left(\left(I-\lambda c^{T}\right) A\right)-\beta P \\
A_{12}=\left(\left(I-\lambda c^{T}\right) A\right)^{T} P\left(I-\lambda c^{T}\right) F \\
A_{13}=\left(\left(I-\lambda c^{T}\right) A\right)^{T} P \sigma \lambda \\
A_{22}=\left(\left(I-\lambda c^{T}\right) F\right)^{T} P\left(I-\lambda c^{T}\right) F-F^{T} F \\
A_{23}=\left(\left(I-\lambda c^{T}\right) F\right)^{T} P \sigma \lambda \\
A_{33}=\sigma^{2} \lambda^{T} P \lambda-\sigma^{2} .
\end{array}\right.
$$

Using Definition of positive definite matrix allows to rewrite (14) as:

$$
\left[\begin{array}{ccc}
A_{11} & A_{12} & A_{13} \\
* & A_{22} & A_{23} \\
* & * & A_{33}
\end{array}\right] \preceq 0, \quad \forall\left[\begin{array}{c}
v \\
\tilde{\omega} \\
\eta
\end{array}\right] \neq 0
$$

This is equivalent to:

$$
\left[\begin{array}{ccc}
-A_{11} & -A_{12} & -A_{13} \\
* & -A_{22} & -A_{23} \\
* & * & -A_{33}
\end{array}\right] \succeq 0, \quad \forall\left[\begin{array}{c}
v \\
\tilde{\omega} \\
\eta
\end{array}\right] \neq 0
$$

Using the explicit notations (15) and doing some manipulations in (17) a BMI (Binear Matrix Inequality) is derived as:

$$
\begin{aligned}
& {\left[\begin{array}{ccc}
\beta P & 0 & 0 \\
* & F^{T} F & 0 \\
* & * & \sigma^{2}
\end{array}\right]-} \\
& -\left[\begin{array}{c}
\left(A^{T}-A^{T} c \lambda^{T}\right) P \\
\left.\left(F^{T}-F^{T} c \lambda^{T}\right) P\right) \\
\lambda^{T} P \sigma
\end{array}\right] P^{-1}\left[\begin{array}{c}
\left(A^{T}-A^{T} c \lambda^{T}\right) P \\
\left.\left(F^{T}-F^{T} c \lambda^{T}\right) P\right) \\
\lambda^{T} P \sigma
\end{array}\right]^{T} \succeq 0
\end{aligned}
$$

Using the Schur complement Boyd et al. (1994), it is equivalent to the following BMI with $\beta, P$ and $Y=P \lambda$ as decision variables:

$$
\left[\begin{array}{cccc}
\beta P & 0 & 0 & A^{T} P-A^{T} c Y^{T} \\
* & F^{T} F & 0 & F^{T} P-F^{T} c Y^{T} \\
* & * & \sigma^{2} & Y^{T} \sigma \\
* & * & * & P
\end{array}\right] \succeq 0
$$

Denote the $P$-radius of the state estimation set at instant $k$ as $d_{k}(x)=\max \left(\left\|x-p_{k}\right\|_{P}^{2}\right)$, where $x \in \hat{X}_{k}$ and const $=\max _{\omega}\|F \omega\|_{2}^{2}$, where $\omega \in \mathbf{B}^{n_{\omega}}$. As 2-norm is a convex function and $W$ is a convex set the term const can be easily computed. Then the condition (9) can be written as $d_{k+1}(x) \leq \beta d_{k}(x)+$ const $+\sigma^{2}$. At infinity, this expression is equivalent to:

$$
d_{\infty}(x)=\beta d_{\infty}(x)+\text { const }+\sigma^{2}
$$

leading to

$$
d_{\infty}(x)=\frac{\sigma^{2}+\text { const }}{1-\beta}
$$

Let us consider an ellipsoid $E=\left\{x: x^{T} P x \leq \frac{\sigma^{2}+\text { const }}{1-\beta}\right\}$ which is equivalent to $E=\left\{x: x^{T} \frac{(1-\beta) P}{\sigma^{2}+\text { const }} x \leq 1\right\}$. To minimize the $P$-radius $\left(d_{\infty}(x)\right)$ of the zonotope, the ellipsoid of smallest diameter must be found (Boyd et al. (1994)). The following EVP (eigenvalue problem) has to be solved to find the values of $P=P^{T} \in \mathbb{R}^{n \times n}$ and $\lambda \in \mathbb{R}^{n}$.

\section{Algorithm 2}

For $\beta=0: 0.1: 1$

$\max _{\tau, P, Y} \tau$

subject to the LMIs

$$
\left\{\begin{array}{l}
\frac{(1-\beta) P}{\sigma^{2}+\text { const }} \succeq \tau I \\
{\left[\begin{array}{cccc}
\beta P & 0 & 0 & A^{T} P-A^{T} c Y^{T} \\
* & F^{T} F & 0 & F^{T} P-F^{T} c Y^{T} \\
* & * & \sigma^{2} & Y^{T} \sigma \\
* & * & * & P
\end{array}\right] \succeq 0}
\end{array}\right.
$$

End

The guaranteed state estimation can be executed in two phases:

- Algorithm 2 is executed to find out the optimized value of $\lambda$;

- For $k=1: N$, Algorithm 1 is executed to compute the state estimation set by using the value of $\lambda$ of the last step in the correction step.

Remark 2: In the problem of LMI optimization of Algorithm 2, the decision variables are: $P=P^{T} \in \mathbb{R}^{n \times n}$, $Y \in \mathbb{R}^{n}$ and $\tau \in \mathbb{R}$. Thus the total number of the scalar decision variables is $\frac{n(n-1)}{2}+n+1=\frac{n^{2}+n+2}{2}$. The dimensions of LMIs $(22)$ are $n^{2} \times n$ and $(2 n+1) \times(2 n+1)$ respectively. 
Remark 3: The prediction step using zonotopes is a simple matrix computation. However, this computation increases the order of the zonotope at each step. To control the domain complexity, a reduction step is implemented to bound a high-order zonotope by a lower-order zonotope by using Property 3.

Remark 4: Some LMI optimizations must be solved to obtain the value of $\lambda$ but this computation is done offline. In the next section, a comparison of the computation time of the new algorithm with the algorithms in Alamo et al. (2005) will be presented to clarify the advantage of the proposed method.

\section{ILLUSTRATIVE EXAMPLE}

Consider the following linear discrete-time invariant system:

$x_{k+1}=\left[\begin{array}{cc}0 & -0.5 \\ 1 & 1\end{array}\right] x_{k}+0.02\left[\begin{array}{c}-6 \\ 1\end{array}\right] \omega_{k}$

$y_{k}=\left[\begin{array}{ll}-2 & 1\end{array}\right] x_{k}+0.2 v_{k}$

with $\left\|v_{k}\right\|_{\infty} \leq 1,\left\|\omega_{k}\right\|_{\infty} \leq 1$. The initial state belongs to the box $3 \mathbf{B}^{2}$. The order of the zonotopes is limited to $m \leq 20$ in the interest of a fast simulation. A simple system of order 2 is chosen to reduce the complexity of computation and to facilitate the graphic visualization.

Figures $(2,3,4)$ show the evolution of the predicted state set and the outer approximation of state estimation set. This outer approximation is rapidly reduced at each iteration. The obtained set is an approximation of the real intersection which will become more and more accurate at each iteration (from Figure 2 to Figure 4).

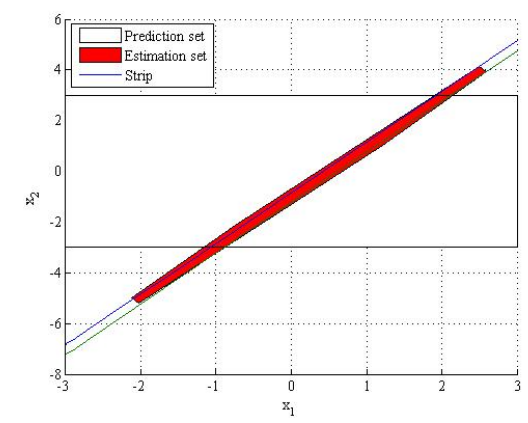

Fig. 2. Intersection $\hat{X}_{k}$ between the predicted state sett $\bar{X}_{k}$ and the measurement $X_{y_{k}}$ at the time instant $k=1$

Figures $(5,6)$ compare the bounds on $x_{1_{k}}$ obtained by the proposed method and the methods developed in Alamo et al. (2005). The dotted lines show the bounds of $x_{1_{k}}$ obtained by the segment minimization algorithm. The dash-dotted lines represent the bounds of $x_{1}$ obtained by the volume minimization algorithm and the solid lines represent the bounds of $x_{1_{k}}$ obtained by the presented algorithm. The stars represent the real state $x_{1_{k}}$ of the system. These points are found in the bounds of $x_{1_{k}}$ confirming that this bound is well estimated.

Figure (7) shows the bound's widths of $x_{1_{k}}$ obtained using different algorithms. If the bound's width of $x_{1_{k}}$ computed by the segment minimization algorithm is considered as 100 , then the bound's widths of $x_{1_{k}}$ computed by the

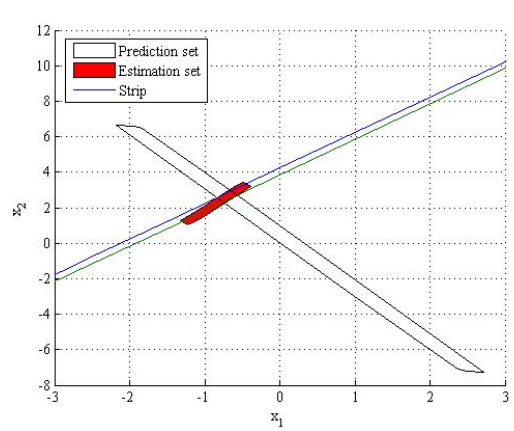

Fig. 3. Intersection $\hat{X}_{k}$ between the predicted state set $\bar{X}_{k}$ and the measurement $X_{y_{k}}$ at the time instant $k=2$

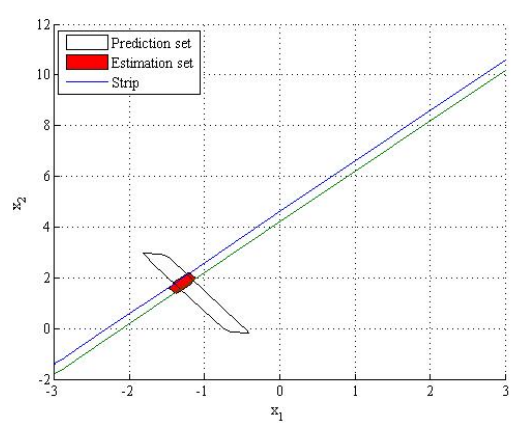

Fig. 4. Intersection $\hat{X}_{k}$ between the predicted state set $\bar{X}_{k}$ and the measurement $X_{y_{k}}$ at the time instant $k=3$

volume minimization algorithm and the new proposed algorithm are compared with the segment minimization method (Figure 7). This figure shows a better performance of the proposed method than the segment minimization method (the bound of $x_{1_{k}}$ is smaller) and the same performance as the volume minimization method after only about 5 iterations.

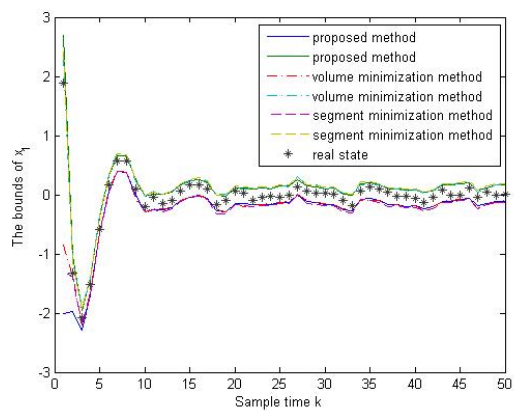

Fig. 5. Bounds of $x_{1}$ obtained by different methods

Table (1) shows the computation time of different algorithms. These results are obtained with an Intel Core 2 Duo E8500 3.16 GHz. The LMI optimizations are solved by using LMI toolbox of Matlab ${ }^{\circledR}$ and the volume minimization problem is solved by the fminsearch function of Matlab. If the time used to solve LMI optimizations is not taken into account, the computation time of this method is the same as the computation of the segment minimization but the performance of estimation is better. Even if the LMI optimizations are taken into account, the computation time is 10 times less than the computation time of the volume minimization method. 


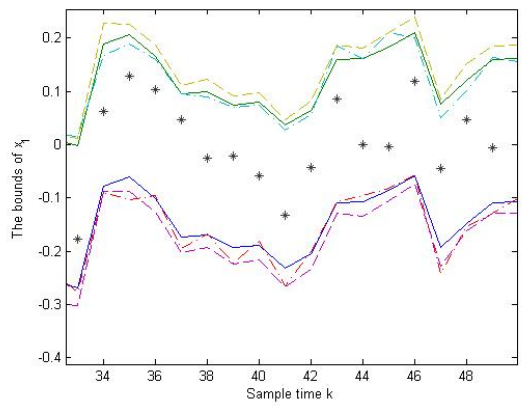

Fig. 6. Bounds of $x_{1}$ obtained by different methods (zoom of Figure 5)

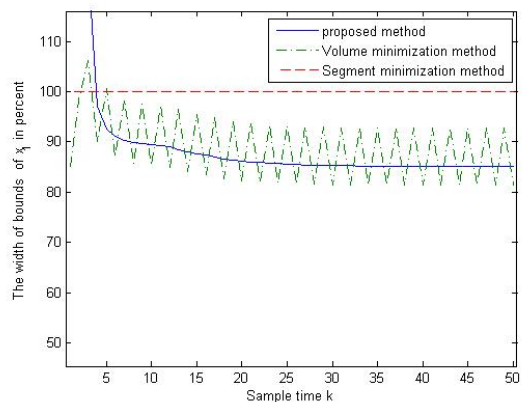

Fig. 7. Comparison of the bound's width of $x_{1}$ obtained by different methods

The segment minimization algorithm has an acceptable performance and a very short computation time (only some computations to obtain $\lambda$ ). The volume minimization algorithm gives a better performance but it needs a longer computation time (251 times more than the segment minimization method) because an optimization problem must be solved at each sample time. The performance of the presented method can be comparable with the performance obtained by the volume minimization algorithm but $\lambda$ is computed at the beginning of the program and at each iteration the value of $\lambda$ does not need to be recomputed. In summary, the proposed algorithm combines the advantage of the volume minimization (performance) and of the segment minimization (computation time). Moreover this algorithm allows to overcome the problem of volume minimization due to a very narrow zonotope.

Table 1. Computation time after 50 sample times

Algorithm

Segment minimization

Presented algorithm (without LMI optimization)

Presented algorithm (with LMI optimization)

Volume minimization

\section{CONCLUSION}

A new guaranteed state estimation method for linear time invariance discrete-time system with bounded noises and bounded perturbations has been proposed. The method computes a set of all the states that are consistent with the measured output, the bounded noise, and the bounded perturbation. The state estimation set is represented by zonotopes and its size is decreased at each sample time by using the presented approach. An example has been provided to clarify and compare the presented algorithm with some existing algorithms. This paper considers a perfectly known state matrix $A$. The extension of the presented algorithm for an uncertain matrix $A$, e.g. $A$ is an interval matrix (i.e. a matrix whose elements are intervals) will be considered in future work.

\section{REFERENCES}

Alamo, T., Bravo, J.M., and Camacho, E.F. (2005). Guaranteed state estimation by zonotopes. Automatica, 41, $1035-1043$.

Althoff, M., Stursberg, O., and Buss, M. (2007). Reachability analysis of linear systems with uncertain parameters and inputs. Proceedings of the 46th IEEE Conference on Decision and Control, 41, 726-732. New Orleans, LA, USA.

Bertsekas, D.P. and Rhodes, I.B. (1971). Recursive state estimation for a set-membership description of uncertainty. IEEE Transactions on Automatic Control, 16(2), 117-128.

Boyd, S., Ghaoui, L.E., Feron, E., and Balakrishnan, V. (1994). Linear Matrix Inequalities in System and Control Theory. SIAM, Philadelphia.

Combastel, C. (2003). A state bounding observer based on zonotopes. Proceedings of European Control Conference. Cambridge, UK.

Durieu, C., Walter, E., and Polyak, B. (2001). Multiinput multi-output ellipsoidal state bounding. Journal of Optimization Theory and Applications, 111(2), 273303.

Guibas, L.J., Nguyen, A., and Zhang, L. (2005). Zonotopes as bounding volume. Proceedings of the Symposium on Discrete Algorithm, 803-812.

Kalman, R.E. (1960). A new approach to linear filtering and prediction problems. Transactions of the ASMEJournal of Basic Engineering, 82(Series D), 35-45.

Kühn, W. (1998). Rigorously computed orbits of dynamical systems without the wrapping effect. Computing, $61,47-67$.

Lalami, A. (2008). Diagonostic et approaches ensemblistes à base des zonotopes. Ph.D. thesis, Université de CergyPontoise.

Schön, S. and Kutterer, H. (2005). Using zonotopes for overestimation-free interval least-squaressome geodetic applications. Reliable Computing Springer, 11, 137-155.

Schweppe, F.C. (1968). Recursive state estimation: Unknown but bounded errors and system inputs. IEEE Transactions on Automatic Control, 13(1), 22-28.

Sorenson, H. (1983). Special issue on applications of kalman filtering. IEEE Transactions on Automatic Control, 28(3), 253-434.

Vicino, A. and Zappa, G. (1996). Sequential approximation of feasible parameter sets for identification with set membership uncertainty. IEEE Transactions on Automatic Control, 41, 774-785.

Walter, E. and Piet-Lahanier, H. (1989). Exact recursive polyhedral description of the feasible parameter set for bounded-error models. IEEE Transactions on Automatic Control, 34(8), 911-915. 